\title{
Grazing Incidence X-ray Optics for Wavelength Dispersive Spectrometers in Micro-Analysis
}

\author{
David OHara
}

Parallax Research, Inc. PO Box 12212, Tallahassee, FL 32317, USA

As Energy Dispersive Spectrometer technology has matured, these detectors have greatly improved resolution and light element performance relative to their predecessors. While it is now routine for an EDS system to be able to detect Boron and sometimes even Be, this improvement in resolution and light element performance has caused the more cumbersome Wavelength Dispersive Spectrometers to become fairly rare. However, when very good resolution, high Peak to Background (P/B), or high count rates for a single element is needed, the only currently available instrument is the WDS system in spite of its difficulty of use and expense. In recent years, advances in X-ray optics have led to a revival of WDS spectrometers in which they can be placed on a wider variety of instruments than ever before and in which they have light element performance far exceeding other spectrometers. Such advances may soon lead to a proliferation of WDS beyond its current niche and into a wide array of scientific and industrial applications.

In this paper, the author attempts to discuss the use of grazing incidence x-ray optics to improve the performance of Wavelength Dispersive Spectrometers and to produce systems with specific desired properties. Grazing incidence optics which utilize the high reflectivity of certain surfaces for x-rays at very small angles (Fig. 1.) include single and multiple reflection geometries from figured surfaces, mono and polycapillary optics, and micro-channel based optics. Because the author is mostly familiar with various types of grazing incidence $\mathrm{x}$-ray optics, this discussion will be concerned with such systems that include grazing incidence beam forming optics used for WDS. A discussion of new types of diffractive optics for x-ray microanalysis cannot be sufficiently covered here except when they are used in combination with grazing incidence x-ray beam forming optics. This discussion includes not only existing WDS systems but also some under development and some concepts for advanced systems.

The paper discusses the advantages, disadvantages, properties and performance of parallel beam WDS as illustrated by Fig.2. relative to conventional WDS. Modifications to the original parallel beam WDS such as the combined single reflection/polycapillary, advanced polycapillary optics, advanced multiple reflection Wolter optics are discussed. Because some parallel beam WDS systems allow fast scanning, there is a discussion of scanning techniques and purpose and "Intelligent Scanning" for identification of elements when possible constituencies are known. Because conventional WDS has some advantages, the use of nested hyperbolic reflectors to either increase the solid collection angle or ease of mounting a conventional WDS is discussed. Last, a concept allowing narrow energy range scanning but very large collection angle using a combination of unusual grazing incidence and curved diffractors is discussed. 


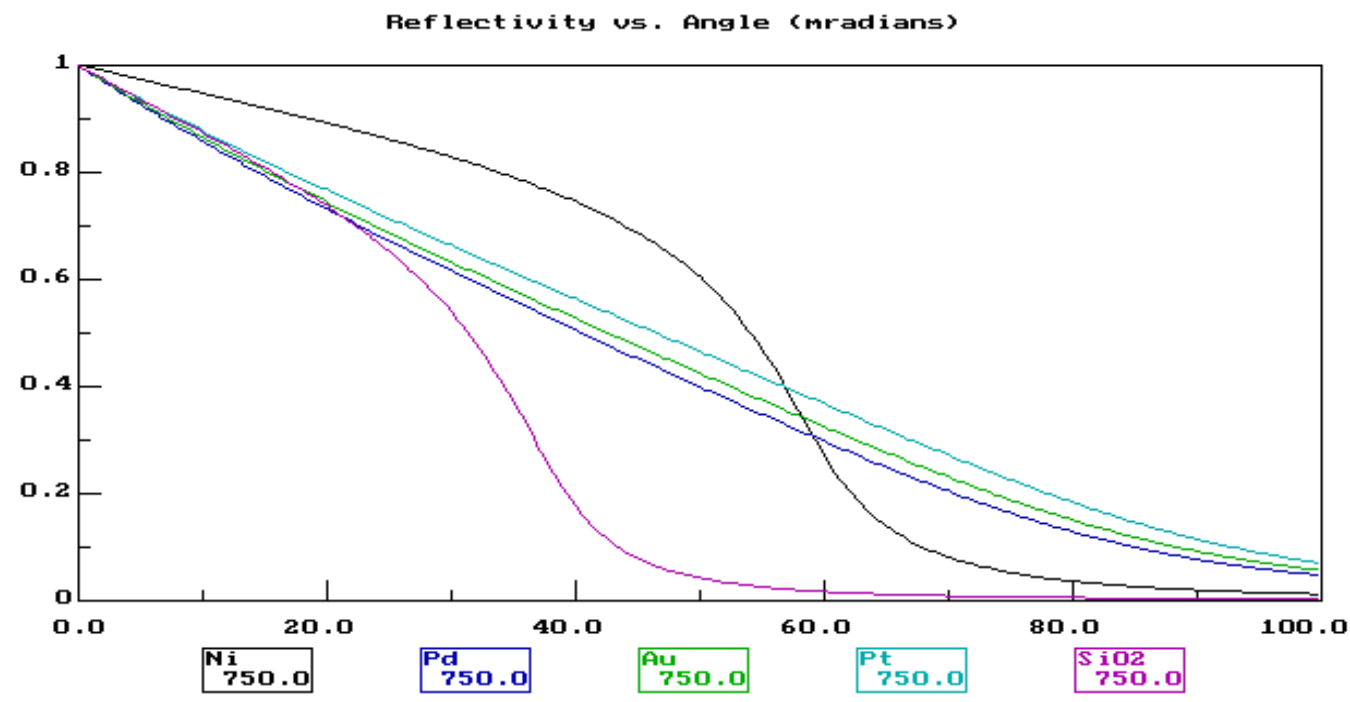

Fig. 1. Reflectivity of several surfaces for $750 \mathrm{eV}$ x-rays as a function of grazing angle.

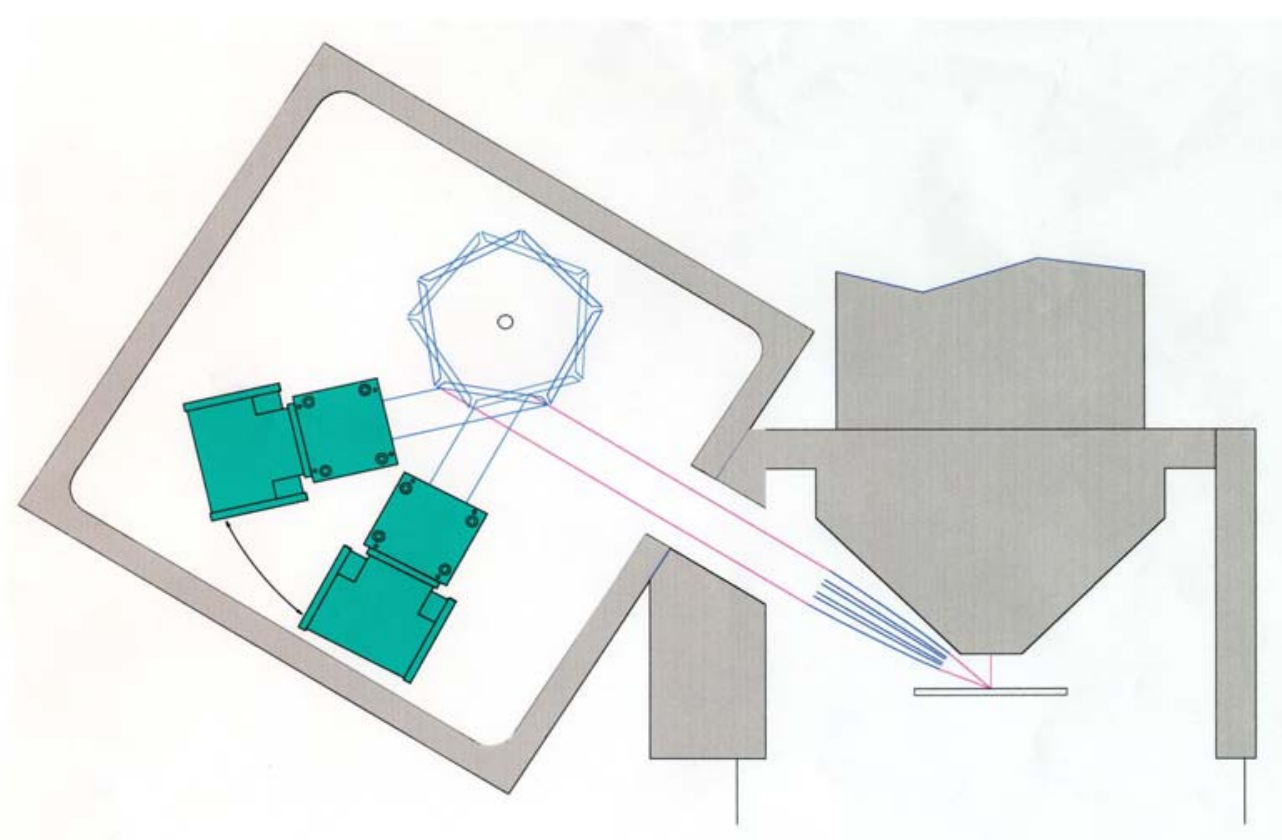

Fig 2. A parallel beam WDS system (In this case, LEXS by Parallax Research, Inc.) on a SEM. The x-ray collimating optics are close to the x-ray source producing a parallel beam of x-rays incident on flat diffractors. A turret contains 5 diffractors reflecting into a detector that moves through $2 \theta$ as the diffractor rotates through $\theta$. 